Produto \& Produção, vol. 11, n. 3, p. 07-18, out. 2010

\title{
Análise não paramétrica de falhas ao longo do calendário para alto-falantes
}

\author{
Vera Lucia Milani Martins \\ Programa de Pós-Graduação em Engenharia de Produção - PPGEP/UFRGS \\ vlmmartins@ producao.ufrgs.br \\ Liane Werner \\ Programa de Pós-Graduação em Engenharia de Produção - PPGEP/UFRGS \\ liane@producao.ufrgs.br
}

A análise de dados de garantia é uma importante fonte de informação para descrever o comportamento dos tempos de falha. Cada produto possui um desempenho único ao ser iniciado o seu uso. O presente trabalho aborda uma modelagem não paramétrica para as falhas ao longo do calendário de alto-falantes, tipo subwoofer com 550 Wrms de potência, item considerado como não reparável. A modelagem das falhas é apresentada conforme duas formas de organização dos dados: a) falhas ao longo do calendário no período de garantia para tamanho de amostras diferentes e b) falhas ao longo do calendário no período de garantia para tamanho de amostras iguais, as estimativas de sobrevivência são geradas através do estimador de Kaplan-Meier. Os resultados mostram que há diferença significativa entre as curvas geradas, e que para cada idade de falha, a curva que melhor representou as falhas é a estimada com tamanhos de amostras iguais.

Palavras-chave: Confiabilidade, alto-falantes, Kaplan-Meier.

The data analysis of products within warranty time is very important to describe the period of failure throughout the calendar of the product. Every product has its own and unique behavior since the start of its use. This paper deals with non-parametric modeling for failures throughout the calendar of subwoofer loudspeakers with $550 \mathrm{Wrms}$ power, an item considered irreparable. The modeling of these failures is presented in two forms to organize the data: a) Failure throughout the calendar within the warranty time, making use of a different quantity for each sample; b) Failure throughout the calendar within the warranty time, making use of the same quantity for each sample. The estimated lifetime is generated by the Kaplan-Meier's estimating. The results show a significant difference among the generated curves. For each failure time, the best representative failure curve is the one estimated with the same quantity for each sample.

Keywords: Reliability, loudspeakers, Kaplan-Meier.

\section{Introdução}

Atualmente, com o mercado mundial globalizado, a corrida pela conquista ao consumidor tem se tornado cada vez mais acirrada. O consumidor atual apresenta um comportamento exigente em relação à qualidade dos produtos que adquire, analisando o chamado custobenefício no momento da aquisição de um bem.

Para assegurar a sua "fatia" de consumidores no mercado, as empresas fabricantes necessitam oferecer produtos com prazos maiores de garantia, tendo em vista que este é um fator importante no processo de decisão de compra. A obtenção de prazos e preços competitivos, a flexibilidade produtiva ou ainda o aumento na qualidade dos produtos, são alguns dos modos de sobrevivência diante dos competidores (WERNER, 1996). Este tipo de oferta só é possível conhecendo o nível de confiabilidade do produto. Quanto maior a confiabilidade de um produto, maior sua vida útil, menor o custo com garantia e maior a satisfação do cliente. Neste contexto, produtos com altos níveis de confiabilidade divulgados através das impressões de consumidor a consumidor e nos prazos de garantia, detêm uma fatia crescente e fidelizada do mercado.

Devido à ação de agentes naturais como, por exemplo, a temperatura, umidade e salinidade, os produtos poderão trabalhar de forma degradada ou falhar em diferentes tempos, motivo pelo qual o cálculo de confiabilidade faz referência a um período de tempo determinado. É preciso salientar que além de fazer referência a um período de tempo, o cálculo da confiabilidade também ressalta a importância do uso adequado do produto, respeitando a finalidade a qual o mesmo se destina. 
Com a complexidade dos produtos e equipamentos produzidos atualmente, torna-se necessária a produção com alto nível de confiança, como por exemplo, equipamentos de telecomunicação. A previsão da confiabilidade na fase inicial do projeto a partir de modelos de falhas padronizados e a realização de testes acelerados de vida nos protótipos são algumas das ferramentas disponíveis para obtenção de uma estimativa inicial da confiabilidade e correção precoce de falhas que só seriam detectadas no uso real do produto, após seu lançamento. Os pequenos fabricantes de equipamentos de alta tecnologia, pressionados pela necessidade de desenvolvimento acelerado de seus produtos e limitados em recursos financeiros e humanos, geralmente desconhecem ou não aplicam estas ferramentas nos estágios iniciais do desenvolvimento e, muitas vezes o resultado é o lançamento de produtos com problemas crônicos de confiabilidade (DILLENBURG, 2005).

Uma vez no mercado, o produto é operado pelos clientes e eventualmente apresenta falhas que resultam no seu retorno ao fabricante, principalmente durante o período de garantia. É exatamente nesse momento que existe uma excelente oportunidade de coleta de informações referentes à sua confiabilidade, que adequadamente registradas e processadas, permitem estimar parâmetros de confiabilidade dos produtos, sem gerar custos excessivos ao fabricante. A monitoração destes parâmetros permite detectar precocemente problemas, prever custos futuros, dimensionar estoques e equipes de manutenção e prover a área de pesquisa e desenvolvimento (P\&D) com informações úteis ao aprimoramento deste e de futuros produtos (DILLENBURG, 2005).

Para as empresas se faz cada vez mais necessária a aplicação de técnicas estatísticas que descrevam a qualidade de seus produtos e indiquem melhorias dos mesmos, não apenas por motivações como redução de custos, ampliação do número de seus consumidores ou determinação de prazos de garantia, mas também por ser uma exigência do mercado que se vale cada vez mais de especificações normativas, como a ISO9000. Entre as técnicas estatísticas utilizadas na indústria como ferramentas da qualidade estão: análise de regressão, sete ferramentas da qualidade e confiabilidade (WERKEMA, 1995).

O dinamismo no lançamento de novos produtos, a necessidade de avaliar o comportamento de processos produtivos e a oportunidade de expressar em números as melhorias de produto trouxe para dentro da indústria de alto-falantes técnicas estatísticas aplicadas como o controle estatístico do processo (CEP), a análise do sistema de medição (MSA) e o estudo de confiabilidade.

De uma maneira geral, a noção de confiabilidade está intuitivamente associada ao grau de certeza que se tem no bom funcionamento de um produto ao longo do tempo.
As indústrias de alto-falantes têm como um de seus principais divulgadores e captadores de novos clientes justamente o grau de certeza de desempenho de seus produtos divulgados pelas impressões dos consumidores. Estas impressões são formadas ao longo do tempo, conforme a utilização do alto-falante adquirido, indicando então, a importância de se conhecer o comportamento das falhas do produto pelo fabricante e como elas ocorrem ao longo do calendário.

Sendo assim, o objetivo principal deste trabalho é descrever a confiabilidade do produto alto-falante do tipo subwoofer ( $550 \mathrm{Wrms}$ ) ao longo do calendário. Para tanto será utilizado o estimador não-paramétrico de Kaplan-Meier, sob duas formas contemplando tamanhos de amostras iguais e diferentes para cada período de garantia disposto ao longo do tempo.

A realização deste estudo traz contribuições para a área de confiabilidade em vários aspectos, entre eles tem-se a apresentação da descrição de duas formas de organização dos dados para cálculos de confiabilidade ao longo do calendário, também o uso de um estimador (KaplanMeier) não usual na área de engenharia de produção, e por fim, apresenta uma aplicação em um setor da indústria brasileira que realiza poucos estudos na área.

A metodologia utilizada para verificar qual das formas trás mais ganhos, consiste em após construir a matriz diagonal dos dados coletados, aplicar o estimador de Kaplan-Meier e comparar os resultados obtidos através da análise de regressão dos resíduos de cada modelagem.

\section{Referencial teórico}

Nesta seção, o objetivo é uniformizar o conhecimento sobre garantia, confiabilidade e alto-falantes apresentando conceitos básicos sobre cada um destes tópicos.

\subsection{Garantia}

Como os prazos de garantia oferecidos pelos fabricantes têm-se tornado um diferencial na aquisição de um produto, muitos estudos são realizados com o intuito de aumentar estes prazos e também para não onerar os custos finais dos produtos, além de buscar qual a probabilidade de um produto fabricado e colocado em risco retornar do cliente, com falha, dentro do período de garantia.

Quando um fornecedor garante um produto, ele assume uma obrigação com o consumidor. Essa obrigação gera custos para o fornecedor através das falhas do produto. Em geral os termos de garantia requerem que o produto seja reparado, que uma reposição seja providenciada, ou que um reembolso em dinheiro seja dado (WERNER, 1996). 
No momento em que é solicitada a garantia de um produto é necessário que o fabricante tenha condições de atender as necessidades legais do cliente (prazo e conformidade de produto) e de satisfação pessoal, procurando atender ao cliente com o mínimo de transtornos, visando manter um vínculo com o consumidor. Ao iniciar a utilização de um produto, o mesmo já está sujeito a falhas ou quebras. Quando estas falhas ou quebras ocorrem durante o período de garantia - momento em que é mais provável que o fabricante seja acionado pelo cliente - as informações registradas para análise são as mais completas, pois em geral o registro é feito por uma equipe treinada e com informações uniformizadas sobre os tipos de falhas e ocorrências.

Com a análise dos dados de retorno de campo é possível detectar, analisar e atuar sobre problemas específicos do produto, proporcionado de modo geral eficácia no que diz respeito às quantidades de retorno, sejam estes problemas gerados por componentes ou montagem.

\subsection{Confiabilidade}

Conforme Ribeiro e Bell (1997), para atingir a confiabilidade é preciso entender o significado deste termo. Segundo Halpern (1978) a confiabilidade está embasada em quatro elementos principais: probabilidade, desempenho, tempo de operação e condições de operação. Estes elementos são identificados na definição utilizada por Dhillon (2007) que descreve a confiabilidade como sendo a probabilidade de um item desempenhar sua função satisfatoriamente de acordo com o período especificado, quando usado sob as condições especificadas. Dhillon (2007) também descreve as falhas como a inabilidade de um item para funcionar dentro das normas especificadas. Sendo assim, em confiabilidade mede-se sempre o tempo de vida até a falha ou quebra do produto.

Considerando as definições supracitadas, é razoável considerar que o tempo de vida é uma variável aleatória e a confiabilidade pode ser interpretada como a freqüência relativa da ocorrência de falhas por unidade de tempo. A função de confiabilidade, simbolizada por $\mathrm{R}(t)$, é probabilidade de sobreviver além do tempo t, dada pela equação (1), conforme Meyer (1983). A taxa de falha dos sobreviventes no tempo té dada pela equação (2), conforme Smith (2005).

$$
\begin{aligned}
& R(t)=\int_{t}^{+\infty} f(x) d x, t \geq 0 \\
& h(t)=\frac{f(t)}{R(t)}
\end{aligned}
$$

onde: $f(t)$ é a função densidade de probabilidade no tempo $t$.
Os itens avaliados podem ser reparáveis ou não reparáveis. Para O'Connor (1995), nos itens reparáveis, confiabilidade é interpretada como a probabilidade de que uma falha não ocorra no período de interesse, onde mais de uma falha pode ocorrer e nos itens não reparáveis, confiabilidade é interpretada como a probabilidade de sobrevivência após um tempo de vida esperado, caso dos produtos considerados por este trabalho.

A fonte do banco de dados para análise da confiabilidade pode ser de testes de vida acelerados realizado em protótipos ou obtida da análise de informações de campo (clientes). Neste trabalho os dados de interesse provêm de informações de retorno de campo.

Em geral, os dados de retorno de campo disponíveis pelas empresas indicam a falha ocorrida dentro do período de garantia, como o tempo de garantia tem um limite pré-estabelecido, os dados observados neste período são considerados censurados.

A literatura trata a censura dos dados como sendo do tipo I, do tipo II ou aleatório. A censura do tipo I é a que diz respeito à censura de tempo, quando se estabelece um tempo para verificar as falhas ocorridas dentro do período. A censura do tipo II trata de censuras de falhas, onde os dados são observados até que ocorra um número determinado de falhas. Já a censura do tipo aleatório ocorre quando um item é retirado do teste antes de um exato período de tempo, sem que tenha ocorrido falha, em geral para observar as condições da peça até aquele momento. De acordo com Santos et. al. (2008), dados de garantia são altamente censurados, já que poucas unidades do equipamento devem falhar durante o período de garantia.

O comportamento das falhas pode ser analisado conforme uma abordagem paramétrica ou uma abordagem não paramétrica. A análise das falhas pode ser realizada considerando que os dados se ajustem adequadamente a alguma distribuição de probabilidade conhecida. Este tipo de ajustamento dos dados é conhecido como análise paramétrica.

Em confiabilidade existem modelos que descrevem adequadamente os tipos de dados, o mais utilizado destes é a distribuição de Weibull, mas também as distribuições Exponencial, Normal e Log-normal, por exemplo, são bastante utilizadas. A abordagem paramétrica já é amplamente explorada nos trabalhos relacionados a falhas de produtos no tempo, como exemplo pode-se citar Werner (1996), Allella et. al. (2001) e Dillenburg (2005). Deste modo, a abordagem a ser utilizada neste trabalho será a modelagem não paramétrica das falhas ao longo do calendário, que será abordada na seqüência. 


\subsection{Modelos não paramétricos da confiabilidade}

A utilização de modelos não paramétricos em confiabilidade tem-se dado principalmente pela facilidade de uso e pelas exigências dos testes estatísticos aplicados apresentarem um nível de exigência mais ameno em relação a suposições atribuídas aos modelos paramétricos, como normalidade e heteroscedasticidade, por exemplo. Conforme Siegel (1975), quando tais suposições não são satisfeitas ou quando a mensuração não possui força semelhante à de uma escala intervalar, a determinação de significancia de afirmações fica extremamente prejudicada.

Existem alguns estimadores não-paramétricos para confiabilidade como as Tábuas de Vida, Estimador de Nelson-Aalen e Kaplan-Meier. Para censuras do tipo aleatório, a estimação via Tábuas de Vida torna-se viciada, sendo esta a sua principal desvantagem. Quando o principal interesse está em taxas de riscos cumulativas o estimador de Nelson-Aalen é um dos mais utilizados (Dillenburg, 2005). Um dos estimadores mais indicados para descrever a confiabilidade ao tratar-se de dados com informações provenientes de durabilidade é o estimador de Kaplan-Meier, além de ser adequado também para estimações com tamanhos de amostras de pequeno e médio porte (Borges et. al., 1996), motivos pelos quais este estimador foi escolhido para ser utilizado nesta análise.

Análises de sobrevivencia realizadas pelo estimador de Kaplan-Meier, apresentado originalmente para dados provenientes da área médica (Kaplan e Meier, 1958), são amplamente utilizadas na área da saúde. Porém, é possivel encontrar algumas aplicações na área industrial para estimação de confiabilidade de produtos como eixos de automóveis (Colosimo et al., 1999) e relés (Abackerli et al., 2007).

O estimador de Kaplan-Meier, também conhecido como limite-produto, é um estimador não paramétrico e não viciado para a função de confiabilidade, sua função $\mathrm{R}(t)$ é estimada conforme equação (3). O estimador da taxa de risco $h(t)$, ou em outras palavras, a taxa de falha dos sobreviventes no tempo té apresentado na equação (4). A Figura 1 mostra graficamente a probabilidade de sobrevivência com intervalo de confiança gerado pelo estimador de Kaplan-Meier e na Figura 2 observa-se o comportamento da taxa de risco também gerada pelo mesmo estimador.

$\hat{R}(t)=\left(\left(n_{1}-d_{1}\right) /\left(n_{1}\right)\right)\left(\left(n_{2}-d_{2}\right) /\left(n_{2}\right)\right)\left(\left(n_{t}-d_{t}\right) /\left(n_{t}\right)\right)$

onde: $\mathrm{n}_{\mathrm{i}}$ é número de itens em risco no tempo i e $\mathrm{d}_{\mathrm{i}}$ é o número de falhas no tempo $t_{i}$.

$$
\hat{h}_{(\mathrm{t})=-\log } \hat{R}_{(\mathrm{t})}
$$

Quando ocorre mais que uma falha no mesmo tempo, essas falhas são consideradas como falhas simultâneas e devem ser consideradas para efeito de cálculo no mesmo intervalo de tempo. O valor de $\mathrm{n} 1$ representa o total de itens colocados em risco no tempo 0 e d 1 no tempo 0 é igual a 0 . Desta forma, a probabilidade de sobrevivência inicial é sempre igual a 1.

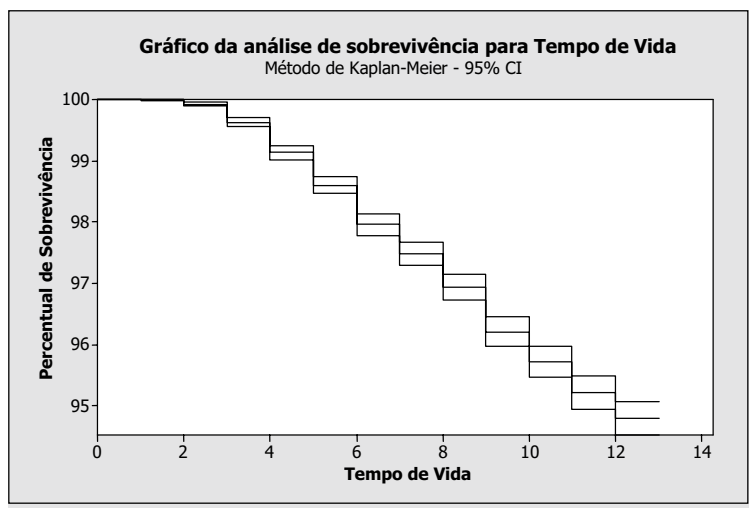

Figura 1 - Gráfico da análise de sobrevivência gerada pelo estimador de Kaplan-Mayer com intervalo de confiança

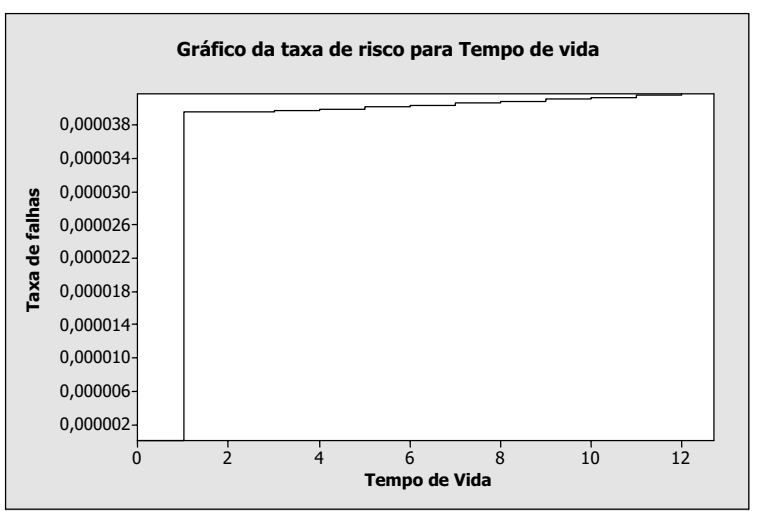

Figura 2 - Gráfico da taxa de risco gerada pelo estimador Kaplan-Meier

\subsection{Alto-falantes}

Os alto-falantes são utilizados para transmitir uma mensagem sonora com seu sinal original amplificado, sua função base consiste em transformar um sinal elétrico em pressão sonora. São considerados como transdutores ou conversores por converter energia elétrica em energia mecânica, que depois é convertida em energia acústica.

Ao adquirir um alto-falante é necessário considerar o que se espera de retorno da conversão sonora descrita acima, considerando, por exemplo, a intensidade (ou volume) e o tom (grave, médio, agudo) que se deseja reproduzir. Em alto-falantes como os quadriaxiais ou triaxiais, são reproduzidos vários tons diferentes, porém perde-se em intensidade. Já nos alto-falantes do tipo subwoofer, considerados neste estudo, reproduz-se apenas um tom (grave) com intensidade elevada. 
Conforme se pode observar na Figura 3 um alto-falante é composto por três partes básicas (i) conjunto magnético: onde é formado o campo magnético, este campo é o que dá força ao alto-falante, motivo pelo qual, nos alto-falantes de maior potência os conjuntos magnéticos são maiores; (ii) conjunto carcaça: é a parte externa do alto-falante, a parte que envolve e protege o conjunto magnético e (iii) conjunto móvel que ao movimentar-se forma uma região de compressão do ar que possibilitará a formação de uma onda sonora.

A primeira conversão de energia (elétrica em mecânica) ocorre quando se coloca a bobina móvel no conjunto magnético e aplica-se uma tensão elétrica alternada nos terminais. Quando a corrente elétrica alternada passa pelo fio da bobina que está imersa no campo magnético, surge uma força eletromagnética. Esta força é perpendicular ao campo e ao fio da bobina e, portanto, movimentará a bobina para cima ou para baixo, como esta é colada a um cone e a uma aranha também se movimentarão protegidos por uma calota e seguros por uma suspensão, formando uma região de compressão e de rarefação que por fim formará então uma onda sonora.

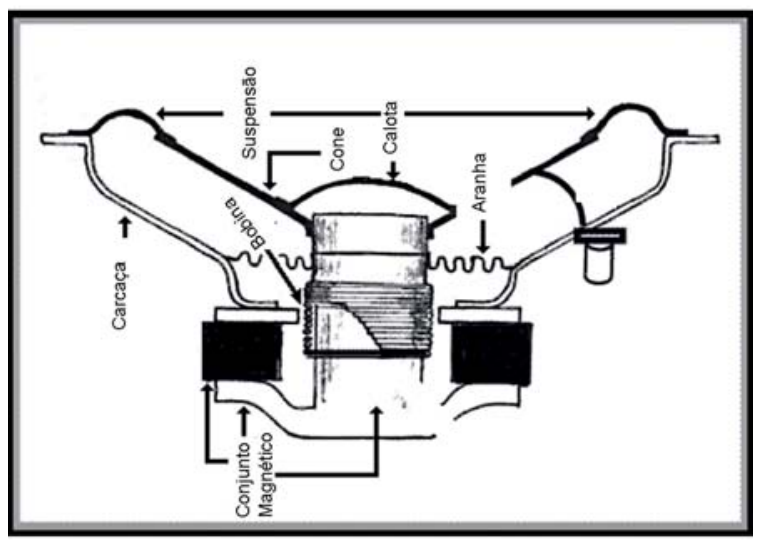

Figura 3 - Vista lateral em corte de um alto-falante

\section{Método aplicado}

\subsection{Estruturação dos dados}

O método utilizado neste trabalho consiste em analisar um mesmo grupo de dados de falhas ocorridas ao longo do calendário, para um produto não reparável, através de duas abordagens diferenciadas, considerando o estimador não paramétrico de Kaplan-Meier.

As observações de falhas de produto ocorrem ao longo tempo mesmo tendo finalizado o período de garantia do mesmo. Incorporar estas informações ao banco de análise representa manter um número maior de dados para os períodos iniciais da estimação de confiabilidade. Ao mesmo passo que manter os registros apenas do período contemplado pela garantia gera uma espera igual ao prazo desta garnatia para a incorporação de novas informações à estimativa da confiabilidade. Desta forma, as abordagens contempladas neste trabalho são:

- Falhas ao longo do calendário no período de garantia para tamanho de amostras diferentes (FLC-AD); - Falhas ao longo do calendário no período de garantia para tamanho de amostras iguais (FLC-AI).

A estruturação das FLC-AD e das FLC-AI é gerada considerando a entrada de falhas ocorridas ao longo do calendário e a entrada de peças colocadas em risco, também dispostas ao longo do calendário. A saída dos dados que constituem as variáveis correspondentes às falhas em cada idade de falha do produto, dispostas ao decorrer do tempo, são as resultantes do somatório de cada uma das diagonais que representa a idade, em meses neste estudo, onde ocorreu a falha.

A configuração de dados contemplando todas as entradas de falhas do produto e de peças colocadas em risco, conforme elas ocorrem ao longo do calendário é exemplificada na Figura 4. A área representada pelo período não observado é o que seria necessário para que as análises, na sua configuração, fossem iguais as que apresentam amostras do mesmo tamanho, porém esta observação não ocorre, pois ao longo do calendário este período ainda não ocorreu.

Para a distribuição dos dados na tabela apresentada pela Figura 4, seguem-se os seguintes passos:

i) Armazenar a quantidade de produção mensal em uma única coluna;

ii) Observar na avaliação de retorno de campo, a data de fabricação do produto;

iii) Armazenar a quantidade de peças com falha no cruzamento entre quantidade produzida e tempo de vida.

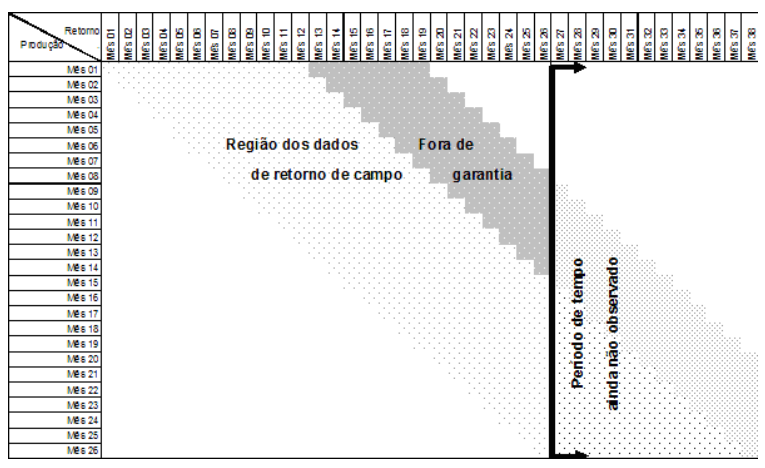

Figura 4 - Dados analisados ao longo do tempo no período de garantia para tamanho de amostras diferentes

A Figura 5 apresenta onde é disposto o corte que delimita tamanhos de amostras iguais. O ponto de corte que difere o tamanho das amostras que compõem 
cada análise é o ponto onde está contemplado, na disposição das linhas, o período completo das falhas ocorridas até último mês de garantia do produto, observando-se, portanto, nesta configuração, todos os itens colocados em risco, e para os quais já se tenha observado cada um dos períodos de falhas ao longo do calendário até a totalidade do período em que é ofertada a garantia do fabricante. O modo de preenchimento da tabela apresentada na Figura 5 segue o mesmo passo a passo descrito anteriormente.

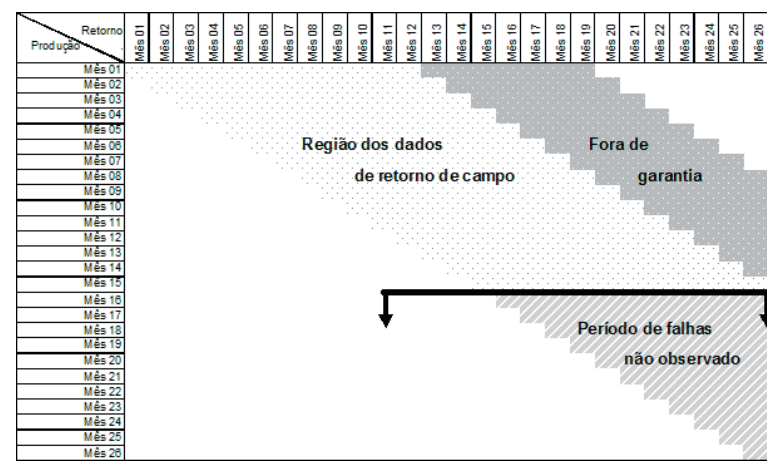

Figura 5 - Dados analisados ao longo do tempo no período de garantia para tamanho de amostras iguais

\subsection{Estrutura da análise}

Para os dados analisados de FLC-AD, a estimação do risco de falha em cada idade do produto, utilizando o estimador de Kaplan-Meier, considerou diferentes quantidades de produtos em risco. Contemplando então, a entrada de novas produções, porém descontando as falhas ocorridas no período imediatamente anterior ao atual, não havendo assim, necessidade de verificar o comportamento de todo período de garantia das falhas mês a mês.

Para os dados analisados de FLC-AI, a estimação do risco de falha em cada idade do produto, utilizando o estimador de Kaplan-Meier, considerou quantidades iguais de produtos em risco, apenas desconsiderando as quantidades de falhas ocorridas no período imediatamente anterior ao atual, não contemplando então a entrada de novas produções e sem a necessidade de verificar o comportamento de todo período de garantia das falhas mês a mês.

As quantidades em risco para FLC-AD em cada mês foram alteradas, uma vez que na idade 1 mês de falhas estavam em risco a quantidade $Y$ de meses de produção, na idade 2 meses de falhas estavam em risco (Y-1) meses de produção, e assim sucessivamente até chegar à idade do período de garantia, em meses de falhas. Já nos dados de FLC-AI, as quantidades de peças em risco

não precisaram ser alteradas por considerarem entradas diferentes nos períodos iniciais de falha.
Depois de delimitadas as formas de análise correspondentes a cada período de falhas dispostas ao longo do calendário, foi obtido o somatório das falhas ocorridas em cada diagonal e ajustada uma quantidade de risco correspondente para cada modo de análise apresentado.

Para o somatório das FLC-AD as quantidades de peças em risco são decrescentes, calculou-se uma quantidade para cada tamanho de amostra utilizado, tantas quantidades em risco foram calculadas quantas foram as entradas de novas peças em risco, conforme Tabela 1. Para os dados das FLC-AI apenas uma quantidade em risco inicial é considerada, utilizando o somatório de cada quantidade de peças em risco por período de tempo observado na análise, conforme apresentado na Tabela 2. No software da análise (MINITAB 15), os dados são apresentados como dados com censura do tipo I, ao longo do tempo, por apresentar as falhas até a totalidade do período de garantia.

Tabela 1 - Exemplo de coleta de dados com tamanho de amostras diferentes em cada idade, com um período de garantia equivalente a 5 meses

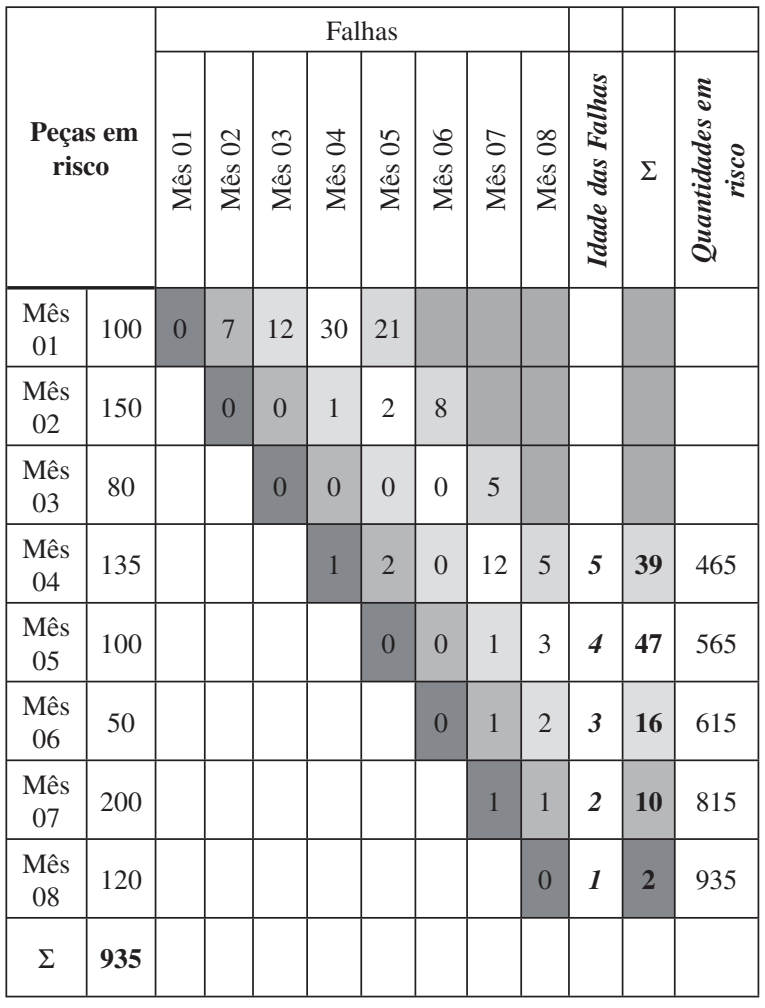


Tabela 2 - Exemplo de coleta de dados com tamanho de amostras iguais em cada idade, com período de garantia equivalente a 5 meses

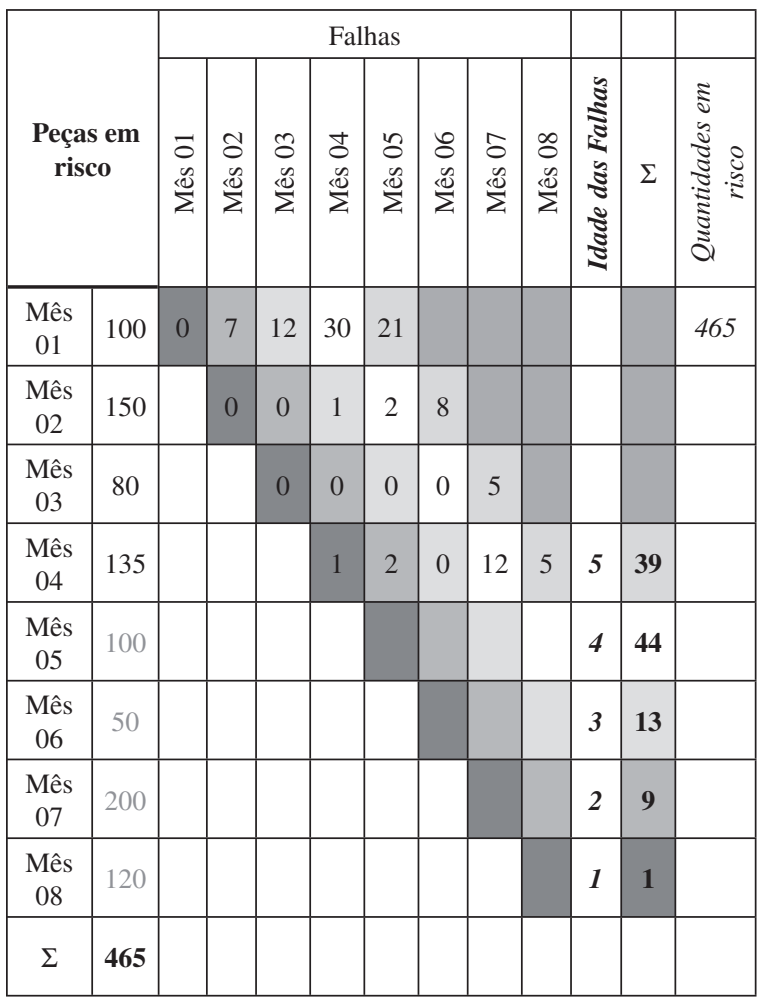

Após aplicar o estimador Kaplan-Meier para as duas formas de estruturação dos dados, será feita uma comparação através da aplicação dos testes de hipótese Log-Rank e de Wilcoxon com a finalidade de verificar se há diferença significativa nos resíduos que compõem cada uma das formas de estimação.

Para complementar os testes executados, será realizada uma análise de regressão simples para cada uma das duas curvas de sobrevivência geradas através dos dados de FLC-AI e de FLC-AD, a fim de indicar qual estruturação dos dados permite maior proximidade entre as falhas esperadas e as falhas obtidas.

\section{Estudo de caso}

A dificuldade na obtenção de bons resultados que descrevam antecipadamente o comportamento de falhas que um alto-falante poderá apresentar após o início de seu uso, está principalmente, no fato de que para altofalantes do tipo subwoofer com potência intermediária (com potencia de $550 \mathrm{Wrms}$ ), as condições de uso especificadas pela empresa fabricante muitas vezes não são respeitadas pelos clientes, não atendendo aos preceitos da confiabilidade. Na Figura 6 uma fotografia do alto-falante modelo subwoofer com potencia de 550 Wrms é apresentada.
A empresa fabricante do alto-falante a ser analisada possui uma preocupação constante com a satisfação de seus clientes, fato pelo qual possui uma política de melhoria continua dos seus produtos e é líder neste segmento do mercado. O dinamismo no lançamento de novas linhas de alto-falantes, uma exigência do mercado atual, não possibilita muitas vezes estudos mais aprofundados que descrevam adequadamente a confiabilidade do produto no seu período inicial de utilização. Uma vez que a empresa fabricante tem como uma de suas principais metas a satisfação do cliente, preocupa-se em estimar falhas ao longo do calendário programando antecipadamente possíveis reposições, dando agilidade ao processo de garantia de seus produtos.

Com o registro histórico dos volumes de produção e das falhas em campo ao longo do calendário estruturou-se uma forma de análise da confiabilidade do produto ao longo do tempo. A conseqüência desta análise consiste na oportunidade de melhorias que podem ser implementadas no processo produtivo ou mesmo nas características do produto. Para as análises dos dados de falhas ao longo do calendário foi utilizado o software Minitab, na versão 15 .

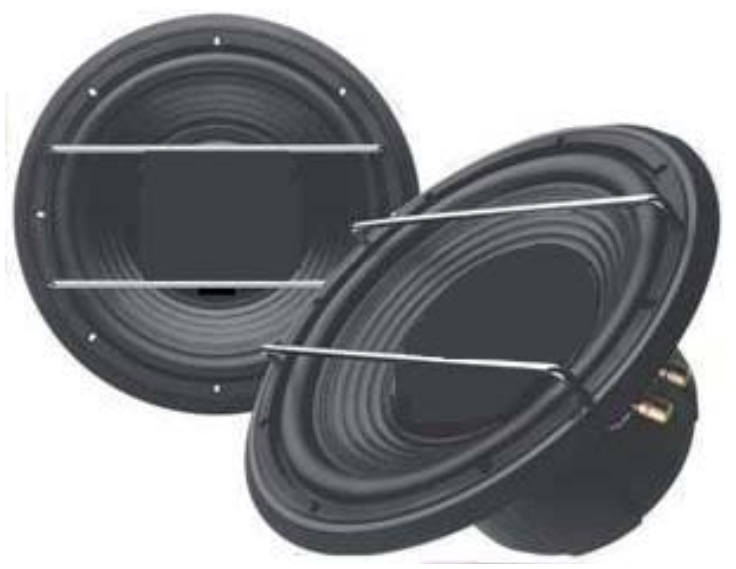

Figura 6 - Alto-falante subwoofer

As tabelas com os dados referentes à disposição mensal das falhas, bem como as análises geradas de acordo com as probabilidades obtidas nas estimativas, utilizadas para realizar as análises de comparação, não serão apresentadas por comporem um conjunto de dados sigilosos da empresa fabricante.

\subsection{Apresentação dos dados}

Para o estudo de caso apresentado a seguir, os dados de análise remetem ao período de 12 meses de garantia oferecidos pelo fabricante e são tratados como itens não reparáveis, já que, quando falham durante o período de garantia são substituídos por unidades novas. 
Os dados coletados são dados de produção, registrados no mês em que foram manufaturados e dados de retorno de campo, que foram agrupados mensalmente de acordo com o mês que retornaram. A planilha de coleta de dados foi estruturada conforme os passos apresentados na seção 3.1: na primeira coluna encontra-se a produção mensal e no encontro das demais colunas com as linhas, os retornos de campo para o respectivo mês de produção e mês de falha.

Como neste trabalho o interesse são as falhas ao longo do calendário os dados a serem analisados consistem da soma das diagonais. A Tabela 3 apresenta a planilha parcial dos dados. O período dos dados obtidos que se refere neste estudo de caso é de 26 meses de um altofalante subwoofer com potencia de $550 \mathrm{Wrms}$.

Tabela 3 - Planilha de coleta de dados dos números de falhas verificados

\begin{tabular}{|c|c|c|c|c|c|c|c|c|c|}
\hline \multirow{2}{*}{\multicolumn{2}{|c|}{$\begin{array}{c}\text { Peças em } \\
\text { risco }\end{array}$}} & \multicolumn{8}{|c|}{ Falhas } \\
\hline & & \multirow{2}{*}{$\begin{array}{l}0 \\
0 \\
\substack{\infty \\
\Sigma}\end{array}$} & \multirow{2}{*}{ 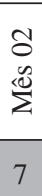 } & \multirow{2}{*}{$\begin{array}{l}0 \\
\sum_{\infty}^{\infty} \\
12\end{array}$} & \multirow{2}{*}{ 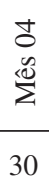 } & \multirow{2}{*}{$\begin{array}{l}n \\
\infty \\
\infty \\
\sum \\
21\end{array}$} & \multirow{2}{*}{ 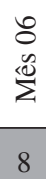 } & \multirow{2}{*}{ 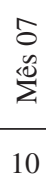 } & \multirow{2}{*}{$\frac{\underset{\infty}{\infty}}{\sum_{\infty}^{\infty}}$} \\
\hline Mês 01 & 1562 & & & & & & & & \\
\hline Mês 02 & 1058 & & 0 & 0 & 1 & 2 & 8 & 20 & 6 \\
\hline Mês 03 & 253 & & & 0 & 0 & 0 & 0 & 5 & 4 \\
\hline Mês 04 & 1270 & & & & 0 & 2 & 0 & 12 & 5 \\
\hline Mês 05 & 1104 & & & & & 0 & 0 & 1 & 3 \\
\hline Mês 06 & 655 & & & & & & 0 & 1 & 2 \\
\hline Mês 07 & 1450 & & & & & & & 1 & 1 \\
\hline Mês 08 & 1229 & & & & & & & & 0 \\
\hline
\end{tabular}

Foram realizadas as duas análises propostas: uma com dados analisados das FLC-AD e outra com dados analisados das FLC-AD. O estimador foi o de Kaplan-Meier, possibilitando assim, uma comparação estimações geradas de ambos os tipos de dados, organizados sempre ao longo do calendário.

\subsection{Análise de FLC-AD}

Para a análise das FLC-AD foram observados os dados disponíveis. Na Figura 4 é possível visualizar as diagonais que possuem tamanhos diferentes, privilegiando as estimações nos períodos iniciais.

O comportamento do somatório gerado em cada diagonal é apresentado na Figura 7. É possível perceber que a quantidade de falhas nas idades prematuras é muito baixa. Isso indica que as oportunidades de melhoria para este tipo de produto estão localizadas no período correspondente a idade adulta do produto, ou seja, as oportunidades de melhoria que causarão maior impacto no aumento da confiabilidade deste produto, correspondem à tomadas de ações referentes a problemas que causaram as falhas ocorridas do quarto ao décimo primeiro mês, aproximadamente.

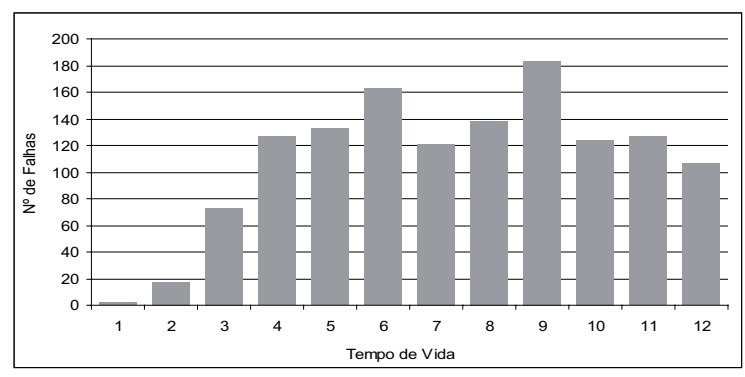

Figura 7 - Comportamento das FLC-AD

Tabela 4 - Estimativas de Kaplan-Meier para dados com diferentes números de peças em risco

\begin{tabular}{|c|c|c|c|c|c|c|}
\hline $\begin{array}{c}\text { Tempo } \\
\text { de vida } \\
\text { (meses) }\end{array}$ & $\begin{array}{c}\text { Risco no } \\
\text { tempo }\end{array}$ & $\begin{array}{c}\text { Falhas no } \\
\text { tempo }\end{array}$ & $\begin{array}{c}\text { Probabilidade de } \\
\text { sobrevivência }\end{array}$ & $\begin{array}{c}\text { Desvio- } \\
\text { padrão }\end{array}$ & $\begin{array}{c}\text { Limite } \\
\text { inferior } \\
\mathbf{9 5 \%} \text { conf }\end{array}$ & $\begin{array}{c}\text { Limite } \\
\text { superior } \\
\mathbf{9 5 \%} \text { conf }\end{array}$ \\
\hline 1 & 25272 & 2 & 0,9999 & 0,00006 & 0,9998 & 1,0000 \\
\hline 2 & 24272 & 18 & 0,9991 & 0,00018 & 0,9988 & 0,9995 \\
\hline 3 & 23850 & 73 & 0,9961 & 0,00040 & 0,9953 & 0,9969 \\
\hline 4 & 22541 & 127 & 0,9902 & 0,00065 & 0,9890 & 0,9916 \\
\hline 5 & 21427 & 133 & 0,9837 & 0,00086 & 0,9820 & 0,9854 \\
\hline 6 & 20391 & 163 & 0,9751 & 0,00108 & 0,9730 & 0,9772 \\
\hline 7 & 19196 & 121 & 0,9677 & 0,00126 & 0,9652 & 0,9701 \\
\hline 8 & 17861 & 139 & 0,9580 & 0,00147 & 0,9551 & 0,9609 \\
\hline 9 & 16174 & 183 & 0,9434 & 0,00177 & 0,9399 & 0,9469 \\
\hline 10 & 15602 & 124 & 0,9346 & 0,00192 & 0,9308 & 0,9384 \\
\hline 11 & 14829 & 127 & 0,9239 & 0,00210 & 0,9198 & 0,9281 \\
\hline 12 & 13601 & 106 & 0,9111 & 0,00238 & 0,9065 & 0,9157 \\
\hline
\end{tabular}


Com a descrição das falhas em cada idade e as quantidades em risco, se estimou a taxa de risco e as probabilidades de sobrevivência do produto com o estimado de Kaplan-Meier. As estimativas de Kaplan-Meier para estes dados são apresentadas na Tabela 4.

A Tabela 4 informa as quantidades em risco (em peças) para um determinado tempo de vida (em meses), as falhas ocorridas em cada tempo de vida (em peças) e a probabilidade de sobrevivência com $95 \%$ de confiança. A probabilidade de um item em risco sobreviver ao mês 1 de vida, com $95 \%$ de confiança, é um valor contido no intervalo de 0,9998 a 1,0000. Tendo sobrevivido ao mês 1 de vida a probabilidade de sobreviver ao mês 2 de vida, com $95 \%$ de confiança, é um valor contido no intervalo de 0,9988 a 0,9995 e assim sucessivamente até o mês 12 de vida que mostra que a probabilidade de um item em risco sobreviver além do período de garantia oferecido pela empresa é um valor contido no intervalo de 0,9066 a 0,9157 .

Seguindo parâmetros de uma política de qualidade da empresa, de satisfação do cliente e de viabilidade financeira, para se considerar um aumento no prazo de garantia deste produto é necessário que a confiabilidade do produto seja ainda melhor. Mesmo com as reclamações de falhas estimadas em aproximadamente $9 \%$ dos produtos colocados em risco, as quantidades efetivas a que correspondem estes $9 \%$, pelo volume de produção é muito alta.

\subsection{Análise de FLC-AI}

Para a análise das FLC-AI, foram observados os dados disponíveis, conforme exemplificado anteriormente na Figura 5, para um período de 12 meses de garantia. Na Figura 5 também é possível visualizar que as diagonais observadas possuem tamanhos iguais, de forma a não privilegiar as estimações em quaisquer dos periodos de observação.

O comportamento do somatório gerado em cada diagonal é apresentado na Figura 8. É possível perceber que a quantidade de falhas nas idades prematuras é muito baixa. Isso indica que as oportunidades de melhoria para este tipo de produto estão localizadas no períodocorrespondente a idade adulta do produto, ou seja, as oportunidades de melhoria que causarão maior impacto no aumento da confiabilidade deste produto, correspondem à tomadas de ações referentes a problemas que causaram as falhas ocorridas do quarto ao décimo primeiro mês, aproximadamente.

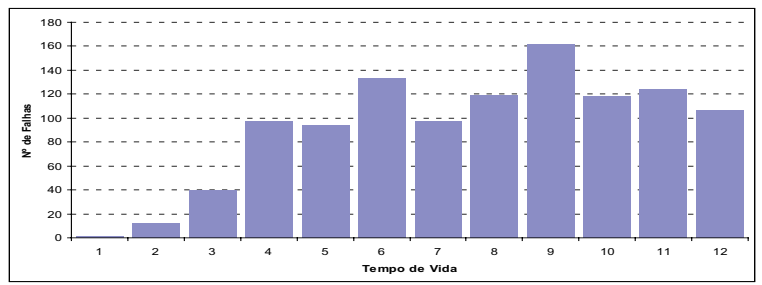

Figura 8 - Comportamento das FLC-AI

As estimativas de Kaplan-Mayer para estes dados são apresentadas na Tabela 5. A Tabela 5 informa as quantidades em risco (em peças) no tempo de vida (em meses), as falhas ocorridas em cada tempo de vida (em peças) e a probabilidade de sobrevivência com $95 \%$ de confiança.

A probabilidade de um item em risco sobreviver ao mês 1 de vida, com $95 \%$ de confiança, é um valor contido no intervalo de 0,9998 e 1,0000. Tendo sobrevivido ao mês 1 de vida a probabilidade de sobreviver ao mês 2 de vida, com $95 \%$ de confiança, é um valor contido no intervalo de 0,9986 e 0,9996 e assim sucessivamente até o mês 12 de vida que mostra que a probabilidade de um

Tabela 5 - Estimativas de Kaplan-Meier para dados com único número de peças em risco

\begin{tabular}{|c|c|c|c|c|c|c|}
\hline $\begin{array}{c}\text { Tempo } \\
\text { de vida } \\
\text { (meses) }\end{array}$ & $\begin{array}{c}\text { Risco no } \\
\text { tempo }\end{array}$ & $\begin{array}{c}\text { Falhas no } \\
\text { tempo }\end{array}$ & $\begin{array}{c}\text { Probabilidade de } \\
\text { sobrevivência }\end{array}$ & $\begin{array}{c}\text { Desvio- } \\
\text { padrão }\end{array}$ & $\begin{array}{c}\text { Limite } \\
\text { inferior } \\
\text { 95\% conf }\end{array}$ & $\begin{array}{c}\text { Limite } \\
\text { superior } \\
\text { 95\% conf }\end{array}$ \\
\hline 1 & 14811 & 1 & 0,9999 & 0,00006 & 0,9998 & 1,0000 \\
\hline 2 & 14810 & 12 & 0,9991 & 0,00024 & 0,9986 & 0,9996 \\
\hline 3 & 14798 & 40 & 0,9964 & 0,00049 & 0,9954 & 0,9973 \\
\hline 4 & 14758 & 97 & 0,9898 & 0,00082 & 0,9882 & 0,9914 \\
\hline 5 & 14661 & 94 & 0,9835 & 0,00104 & 0,9814 & 0,9855 \\
\hline 6 & 14567 & 133 & 0,9745 & 0,00129 & 0,9721 & 0,9770 \\
\hline 7 & 14434 & 97 & 0,9679 & 0,00144 & 0,9651 & 0,9708 \\
\hline 8 & 14337 & 119 & 0,9599 & 0,00161 & 0,9568 & 0,9631 \\
\hline 9 & 14218 & 162 & 0,9490 & 0,00180 & 0,9454 & 0,9525 \\
\hline 10 & 14056 & 118 & 0,9410 & 0,00193 & 0,9372 & 0,9448 \\
\hline 11 & 13938 & 125 & 0,9326 & 0,00205 & 0,9285 & 0,9366 \\
\hline 12 & 13813 & 106 & 0,9254 & 0,00215 & 0,9212 & 0,9296 \\
\hline
\end{tabular}


item em risco sobreviver além do período de garantia oferecido pela empresa é um valor contido no intervalo de 0,9212 e 0,9296 .

Seguindo parâmetros de uma política de qualidade da empresa, de satisfação do cliente e de viabilidade financeira, para se considerar um aumento no prazo de garantia deste produto é necessário que a confiabilidade do produto seja ainda melhor. Mesmo com as reclamações de falhas estimadas em aproximadamente $8 \%$ dos produtos colocados em risco, as quantidades efetivas a que correspondem estes $8 \%$, pelo volume de produção é muito alta.

\subsection{Comparação dos resultados}

Como foram realizadas duas análises dos dados de retorno de campo do alto-falante do tipo subwoofer 550 Wrms, uma para os dados analisados FLC-AI e outra para os dados FLC-AD, aplicou-se testes de hipótese com a finalidade de verificar se há diferença significativa nos resíduos que compõem cada uma das formas de estimação.

Os testes aplicados para comparação entre as curvas de sobrevivência geradas pelos dois formatos de organização dos dados indicaram que existe diferença significativa entre estas curvas. O teste Log-Rank, que compara o número atual e o número esperado de falhas entre as duas curvas para cada falha no tempo, mostrou que a diferença encontrada entre as curvas é significativa, (p-value $<0,001)$. O teste Wilcoxon, que utiliza o número de sobreviventes para ponderação em cada ponto, mostrou que a diferença encontrada entre as curvas também é significativa ( $p$-value $<0,001$ ). Para maiores detalhes sobre os testes de Log-Rank e Wilcoxon veja o trabalho apresentado por Bohoris (1997).

A Figura 9 mostra as curvas de sobrevivência e seus intervalos de confiança, gerados através da análise dos dados das FLC-AI e das FLC-AD. Nesta Figura também é possível visualizar a diferença encontrada nos testes, com a curva que representa os dados que foram analisados utilizando tamanhos de amostras iguais, apresentando uma taxa de sobrevivência maior do que a curva que representa aos dados que utilizaram tamanhos de amostra diferentes.

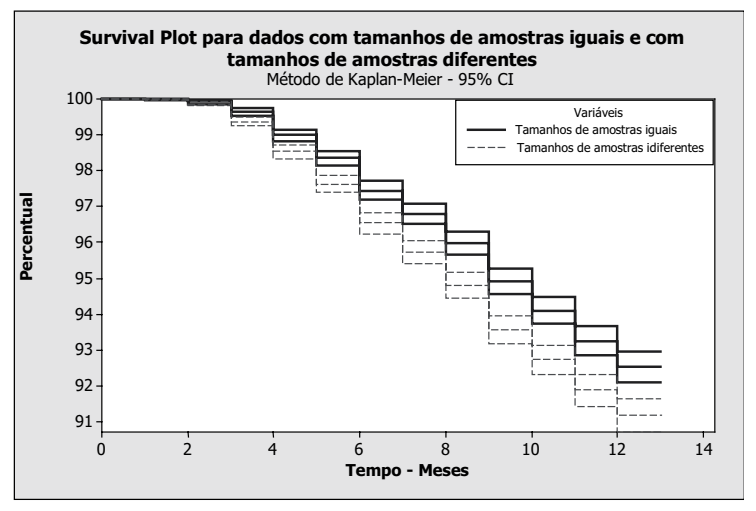

Figura 9 - Comportamento das curvas de sobrevivência e seus intervalos de confiança, geradas através da análise de dados de FLC-AI e de FLC-AD.

Para complementar os testes executados e indicar qual estruturação dos dados permite maior proximidade entre as falhas esperadas e as falhas obtidas, foi realizada uma análise de regressão simples para cada uma das duas curvas de sobrevivência geradas através dos dados de FLC-AI e de FLC-AD. Foi considerado como variável resposta o valor encontrado no somatório das diagonais que continham as quantidades de falhas (em peças) de cada idade do alto-falante em estudo, e considerado como valor predito, o valor esperado para o somatório da diagonal de falhas (em peças) de cada idade do alto-falante de acordo com as estimativas de probablilidade de falhas geradas através das curvas de sobrevivência para cada período.

As análises dos resíduos, apresentada nas Figuras 10 e 11 indicam que a curva que descreve de forma mais acurada o comportamento dos dados acumulados no período estudado é a dos dados organizados ao longo do calendário que contempla o mesmo tamanho de amostra para todas as idades, tal resultado corrobora as conclusões obtidas com os gráficos apresentados pelo testes Log-Rank e Wilcoxon.

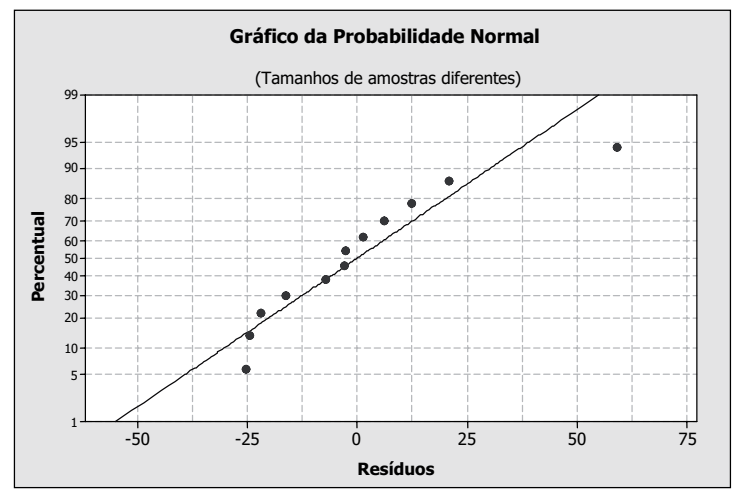

Figura 10 - Resíduos para os dados de FLC-AD 


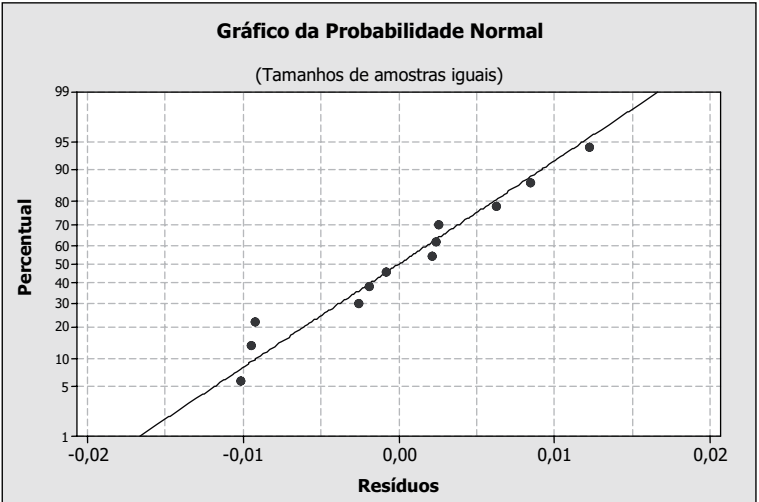

Figura 11 - Resíduos para os dados de FLC-AI

\section{Conclusões}

Este trabalho teve como proposta descrever a confiabilidade do produto alto-falante do tipo subwoofer com potência de $550 \mathrm{Wrms}$ ao longo do calendário através do estimador não paramétrico de Kaplan-Meier.

Os fatos que motivam e tornam necessária a modelagem das falhas de um produto são vários: a programação de reposição de quebras, a adequação das equipes de assistência técnica, o dimensionamento do tempo de garantia que pode ser ofertado ao consumidor.

As alternativas para a modelagem das falhas do altofalante subwoofer 550 Wrms ao longo do calendário, propostas neste trabalho, foram: análise das falhas ao longo do calendário no período de garantia para tamanho de amostras iguais e para tamanhos de amostras diferentes.

Foi possível verificar que, em estudos de confiabilidade que utilizem dados provenientes de falhas no campo de alto-falantes do tipo subwoofer, com potência $550 \mathrm{Wrms}$ e produzido nas mesmas condições que os contemplados neste estudo de caso, a curva de sobrevivência gerada pelo estimador de Kaplan-Meier, com a organização dos dados que utilizaram tamanhos de amostras iguais, quando comparada à curva de sobrevivência gerada através dos dados analisados com tamanhos de amostras diferentes para cada idade do produto, apresentou diferença significativa.

A organização dos dados que considera tamanhos de amostras maiores nas idades iniciais, para este tipo de produto, pode não ter sido eficiente, pois pela sua estrutura de montagem, a temperatura e umidade do ar podem constituir influência importante no resultado final do produto, podendo então existir correlação entre o período de produção e a quantidade de retorno de campo. A melhor avaliação para descrever a curva de sobrevivência através de retorno de campo, é quando as amostras são do mesmo tamanho para todas as idades de sobrevivência, já que, com amostras de tamanhos diferentes contemplam-se períodos que envolvem condições diferentes para cada idade.

Para a empresa fabricante, a importância de conhecer a confiabilidade do produto e a descrição da curva de sobrevivência disposta ao longo do calendário, é que possibilitará a implementação de melhorias no produto e a possibilidade de, no futuro, ter um referencial para comparação da efetividade das melhorias aplicadas.

Fica como sugestão para trabalhos futuros, a análise de dados de produtos deste tipo, considerando outros grupos de potência, a verificação de períodos de sazonalidade nas quantidades de retorno de campo. Pode-se realizar a aplicação de modelagem paramétrica para este tipo de produto. A comparação de uma modelagem paramétrica com uma modelagem não paramétrica, apresentada neste trabalho, também representa uma oportunidade para trabalhos futuros.

\section{Referências}

ABACKERLI, A. J.; PAPA, M. C. O.; MIGUEL, P. A. C.; SASSERON, P. L. Análise da incerteza experimental na determinacão da vida usando ensaio acelerado. Gestão e Produção, São Carlos, v. 14, n. 1, p. 69-81, jan/abr. 2007.

ALLELLA, F.; CHIODO, E. ; LAURIA, D. ; PAGANO, M.. Negative Log-Gamma distribution for data uncertainty modelling in reliability analysis of complex systems - Methodology and robustness. International Journal of Quality \& Reliability Management; Volume: 18 Issue: $3 ; 2001$

BOHORIS, G.A. Calculating the comparative twosample tests for censored reliability data. International Journal of Quality \& Reliability Management; Volume: 14 Issue: $1 ; 1997$.

BORGES, W.S.; COLOSIMO, E. A.; FREITAS, M. A. Métodos Estatísticos e Melhoria da Qualidade: Construindo Confiabilidade em Produtos. $12^{\circ}$ SINAPE ABE - Associação Brasileira de Estatística; 1996.

COLOSIMO, E. A. C.; PONTEL, A. G.; FREITAS, M. A. Avaliando a Confiabilidade de Eixo Traseiro de Automóvel Utilizando um Experimento Planejado. In: ENCONTRO NACIONAL DE ENGENHARIA DE PRODUÇÃO, 19, Rio de Janeiro, 1999. Anais... Rio de Janeiro: Sociedade Brasileira de Engenharia de Produção, 1999.

DHILlON, B.S. Applied Reliability and Quality: Fundamental, methods and Procedures; Springer, 2007. 
DILLENBURG, M. R. Estimativas de confiabilidade de produto a partir das contagens mensais de vendas e falhas ao longo do período de garantia. Dissertação de mestrado em engenharia de produção, UFRGS, Porto Alegre, 2005.

HALPERN, S. The Assurance Science. An introduction to quality control and reliability. New Jersey. USA. 1978.

KAPLAN, E. L.; MEIER, P. Nonparametric estimation from incomplete. Journal of the American Statistical Association, v. 53, n. 282, p.457-481, 1958.

MEYER, P. Probabilidade: Aplicações à estatística. Livros técnicos e científicos editora. 1983.

O'CONNOR, Patrick D. T. Practical reliability engineering. 3ed. Wiley, 1995.

RIBEIRO, J. L. D.; BELL, J.O.C. Avaliação de prazos de garantia. EGATEA; v25, n³ p.105-115, Maio/ Junho 1997.

SANTOS, G.T.; FOGLIATO, F.S.; RIBEIRO. J.L.D.; RAUSAND, M. Aplicação de um modelo de riscos concorrentes na análise de confiabilidade de dados de garantia. Produto \& Produção, vol. 9, n. 3, p. 55-65, out. 2008

SIEGEL, S. Estatistica não-paramétrica para as ciencias do comportamento. 2. ed. Porto Alegre: Artmed, 2006. 448 p.

SMITH, D. J. Reliability, maintainability and risk. 7 ed. Ed. Elsevier, 2005.

WERKEMA, M. C. C. TQC Gestão pela qualidade total: Ferramentas estatísticas básicas para o gerenciamento de processos. Fundação Cristiano Ottoni, Escola de Engenharia da UFMG, v.2, 1995.

WERNER, L. Modelagem dos tempos de falha ao longo do calendário. Dissertação de mestrado em engenharia de produção. UFRGS, Porto Alegre, 1996. 